\title{
Prospects for a MRSA vaccine
}

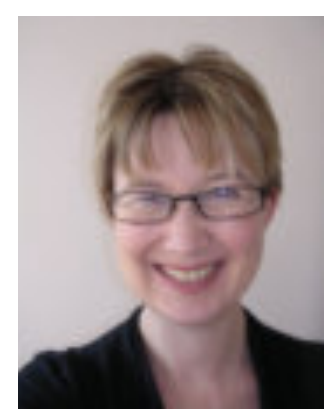

Jodi A Lindsay Centre for Infection, Department of Cellular \& Molecular Medicine, St George's, University of London, Cranmer Terrace, London SW17 ORE, UK Tel.: +44208725 0445; Fax: +44 2087253487 ; jlindsay@sgul.ac.uk
'... without more basic research into host immunity, a vaccine seems a long way off, if possible at all.'

\section{Why do we need a vaccine?}

Methicillin-resistant Staphylococcus aureus (MRSA) is a common cause of infection in hospitals and incidence is linked in the public's mind with hospital cleanliness. In the UK, MRSA causes approximately 7000 cases of bacteremia every year, with a mortality rate of approximately $25 \%$. It probably causes $10-20$-times more infections that are not reported, predominantly wound infections and those associated with intravascular access devices.

But MRSA is only the tip of the iceberg. The real problem is $S$. aureus itself, which causes another 11,000 bacteremias with only a slightly lower mortalilty rate. Again, an enormous number of unreported infections also occur. It is well documented that the UK has one of the highest incidences of MRSA per $S$. aureus infection in Europe (40\%); however, it is difficult to know how to interpret this information since we don't know the incidence of $S$. aureus infections in other countries.

Ideally, S. aureus infections are treated with flucloxacillin, but MRSA are resistant and treated with vancomycin. The first cases of fully vancomycin-resistant MRSA have recently been described. Although several new antimicrobials have been released or are due for release, resistance is already described. Not only do we rely on antimicrobials for treatment, but we are heavily dependent on them for prophylaxis, so that as resistant strains evolve we expect to see infection rates increase. Recent high profile attempts to improve infection control strategies to reduce MRSA incidence in the UK have largely been ineffective.

Not only is $S$. aureus a problem in hospitals, but MRSA strains carrying genes for the Panton-Valentine leukodicin (PVL) are spreading through healthy populations, particularly in the USA [1]. They cause increasing numbers of severe skin and soft tissue infections, as well as fatal hemolytic pneumonia and shock.
High-risk groups are those in the military, sports teams, prisoners, intravenous drug users and children.

A vaccine to $S$. aureus infection would therefore be a useful therapeutic and commercially very attractive. However, it is not obvious who should receive a vaccine, especially when tackling hospital infection. Heathcare workers may be a logical step, although this is unlikely to prevent spread of $S$. aureus via unwashed hands. Immunocompromised patients often respond poorly to vaccines. Colonized patients may be attractive targets, yet few hospitals routinely screen patients on admission to determine who are the carriers, and thus they are rarely offered prophylactic antibiotics that might be just as effective as a vaccine. Despite these limitations, several companies have invested heavily in $S$. aureus vaccine research.

\section{'A vaccine to $S$. aureus infection would therefore be a useful therapeutic and commercially very attractive.'}

\section{Progress so far}

Several antigens have been successfully used to vaccinate mice, which were then protected from disease. These have included capsule, clumping factors (clf) A and B, polysaccharide intracellular adhesin, DNA, iron surface determinant-B and alpha toxin. Some groups have proposed using surface proteins that have subsequently been shown to vary between strains [2], such as serine aspartate repeat proteins, fibronectin-binding proteins and collagenbinding proteins. These targets are less attractive, as an effective vaccine will need to contain the full spectrum of variant types of these proteins. Others have used whole cell extracts, including some that have focused on growing the bacteria in conditions mimicking those found in vivo, as S. aureus are known to alter surface structure under these conditions. Still others have used specific toxins, such as toxicshock syndrome toxin-1 or enterotoxin B, although these toxins are only carried by a small proportion of isolates, and thus are unlikely to have widespread efficacy. 
Two of the most promising candidates, capsule and clfA, have progressed to Phase III clinical trials in humans. The capsule vaccine was developed by Nabi and called StaphVax. It consisted of the two types of $S$. aureus capsules, type 5 and type 8 , combined with pseudomonas exotoxin A toxoid. A Phase III trial in 1800 renal dialysis patients over 73 centers demonstrated elevated antibodies to capsule, but only marginal protection from S. aureus infection. A subsequent Phase III trial failed to show protection. The clfA vaccine was developed by Inhibitex and called Veronate ${ }^{\circledR}$. The vaccine consisted of sera from patients with elevated clfA titers, and the Phase III trial targeted sepsis in low birth-weight babies. It too failed to show protection from $S$. aureus infection. Unfortunately, the failure of these expensive and complex clinical trials may prevent future commercial investment into staphylococcal vaccines.

'...we are all exposed to S. aureus

frequently, and only a small percentage

of the population develops severe infection requiring treatment.'

An alternative strategy to vaccinate against nasal carriage of $S$. aureus has been tested in mice. S. aureus antigens clfB and iron-responsive surface determinants $\mathrm{A}$ and $\mathrm{H}$ have shown promise. However, no trials in humans have so far been published. It is not known if humans colonized with $S$. aureus have different antibodies to those not colonized, or whether antibody is protective. Furthermore, there is some data to suggest $S$. aureus colonization is beneficial to the host, and reduces the severity of subsequent $S$. aureus infection.

\section{What has gone wrong?}

$S$. aureus has a complex interaction with the human host that is not well understood. Of the healthy population, $25 \%$ carry $S$. aureus in their nose all of the time, and another $50 \%$ intermittently. We have all had minor wound infections due to $S$. aureus that lead to inflammation and pus formation, but these are generally controlled by a polymorphonuclear (PMN) lymphocyte response and treatment is unnecessary. Thus, we are all exposed to $S$. aureus frequently, and only a small percentage of the population develops severe infection requiring treatment. These patients are typically the elderly, the very young and the immunocompromised, particularly diabetics. However, occasionally healthy hosts develop severe and life-threatening infection, including those caused by infection with the PVL-positive strains. But even with these strains, only a small percentage of patients exposed will develop disease.

'Why did the vaccines work in the mouse models?

Vaccines work by boosting a protective memory B-cell and antibody response. However, patients who have recovered from a severe $S$. aureus infection often have a substantial antibody response, but are not protected from further infection. If anything, they are typically more susceptible. This is consistent with studies in wild-type versus B-cell-deficient mice, which are equally susceptible to $S$. aureus infection [5]. Thus, antibodies may not be important in the prevention of $S$. aureus infection.

So why did the vaccines work in the mouse models? At this point it is not known, but perhaps there are intrinsic differences between the mouse and human response to $S$. aureus. It is also worth noting that some of the mouse infection models required large numbers of bacteria, overwhelming normal immune reactions. This does not mimic how human $S$. aureus disease develops, which is likely due to relatively small amounts of bacteria introduced directly into a wound or catheter site. Perhaps disease is caused by failure to target and clear relatively small numbers of bacteria by the innate immune response, specifically PMNs, and opsonization may not be dependent on antibody.

Since healthy humans are thought to rely on PMN for clearing $S$. aureus, perhaps we should be focusing more on this response in our efforts to boost immune mediated response to $S$. aureus. However, only a modest community of researchers and funding are focused on these issues. The interactions between $S$. aureus and the human host are complex and currently elusive, and without more basic research into host immunity, a vaccine seems a long way off, if possible at all. 


\section{Bibliography}

1. Moran GJ, Krishnadasan A, Gorwitz RJ et al:: EMERGEncy ID Net Study Group. Methicillin-resistant $S$. aureus infections among patients in the emergency department. N. Engl. J. Med. 355(7), 666-674 (2006).

2. Lindsay JA, Moore CE, Day NP et al.: Microarrays reveal that each of the ten dominant lineages of Staphylococcus aureus has a unique combination of surface-associated and regulatory genes. J. Bacteriol. 188(2), 669-676 (2006).
3. Shinefield H, Black S, Fattom A et al:: Use of a Staphylococcus aureus conjugate vaccine in patients receiving hemodialysis. $N$. Engl. J. Med. 346(7), 491-496 (2002).

4. Capparelli EV, Bloom BT, Kueser TJ et al:: Multicenter study to determine antibody concentrations and assess the safety of administration of INH-A21, a donor-selected human Staphylococcal immune globulin, in low-birth-weight infants. Antimicrob. Agents Chemother. 49(10), 4121-4127 (2005).
5. Gjertsson I, Hultgren OH, Stenson M, Holmdahl R, Tarkowski A: Are B lymphocytes of importance in severe Staphylococcus aureus infections? Infect. Immun. 68(5), 2431-2434 (2000).

\section{Affiliation}

- Jodi A Lindsay Centre for Infection, Department of Cellular \& Molecular Medicine, St George's, University of London, Cranmer Terrace, London, SW17 ORE, UK

Tel.: +44 2087250445 ;

Fax: +44 2087253487 ;

jlindsay@sgul.ac.uk 